\title{
Short Dental Implants: Its Rationale for Use
}

\author{
${ }^{1}$ Mohammed Shammas, ${ }^{2}$ SK Shakeel
}

\begin{abstract}
Atrophy of alveolar bone in the posterior part of the mouth is routinely encountered in clinical practice. The present treatment modality to replace the missing teeth with an implant-retained fixed partial denture includes sinus bone grafting in the maxilla and onlay bone graft in the mandible. This type of treatment is invasive and requires more time and cost. Short dental implants are used as an alternative treatment modality to bone grafting procedures. Short implants could provide equivalent results to those of longer implants if certain principles are followed. The present article reviews the current literature on the use of short implants and discusses the biomechanical considerations when using short implants.
\end{abstract}

Keywords: Short dental implants, Implant length, Endosseous, Sinus floor elevation, Bone grafting.

How to cite this article: Shammas M, Shakeel SK. Short Dental Implants: Its Rationale for Use. Int J Oral Implantol Clin Res 2014;5(2):50-54.

Source of support: Nil

Conflict of interest: None

\section{INTRODUCTION}

Osseointegrated dental implants are an effective alternative in the rehabilitation of partial or completely edentulous patients. ${ }^{1}$ Placement of dental implants is subject to anatomical limitations. ${ }^{2}$ These limitations are more common in the posterior regions of the maxilla and the mandible. ${ }^{3}$ Over the years, many treatment modalities have been proposed to overcome the anatomical limitations of the bone present for implant placement. Several surgical interventions for bone augmentation have been suggested, including bone grafts, guided bone regeneration, distraction osteogenesis, sinus floor elevation, mandibular nerve transposition, and the use of tilted or zygomatic implants. These techniques imply greater morbidity, longer treatment times and higher costs. ${ }^{4}$ Short dental implants have been suggested as another choice for the prosthetic treatment of resorbed alveolar ridges.

\footnotetext{
${ }^{1}$ Associate Professor, ${ }^{2}$ Specialist

${ }^{1}$ Department of Prosthodontics, Ibn Sina National College for Medical Studies, Jeddah, Saudi Arabia

${ }^{2}$ Department of Prosthodontics, Gulf Medical College, Ajman United Arab Emirates
}

Corresponding Author: Mohammed Shammas, Associate Professor, Department of Prosthodontics, Ibn Sina National College for Medical Studies, Jeddah, Saudi Arabia, e-mail: shammasm@ rediffmail.com
However, longer implants have always been considered more reliable due to both an improved crown-to-implant ratio and a greater surface area available for osseointegration, which dissipates the occlusal forces. The introduction of modified implant designs and microstructured implant surfaces that enhance the integratable surface area could help to compensate for the adverse effects of decreasing the implant length, so as to maintain the extent of the boneimplant interface. ${ }^{5}$

There is no consensus in the literature on the definition of a short implant. Some authors consider $10 \mathrm{~mm}$ the minimal length for predictable success; thus, they consider any implant less than $10 \mathrm{~mm}$ in length as short (Morand \& Irinakis 2007). Others defined an implant length of $10 \mathrm{~mm}$ also as a short implant (Das Neves et al 2006). Because an implant can be placed at different levels, a short implant has also been defined as an implant with a designed intra bony length of $8 \mathrm{~mm}$ or less. ${ }^{6-8}$

\section{Rationale for Short Implant Length}

Implant length is generally selected according to the maximum amount of bone height present at the recipient site. This is based on the principle that longer implants provide better primary stability and a favorable distribution of occlusal forces due to an increased total surface area. However, an important difference exists between total surface area and the functional surface area. Total surface area represents the overall surface area of the implant, while a functional surface area represents the area that transfers the compressive and tensile loads to bone and does not include the passive portion of the implant. ${ }^{9}$ The biomechanical rationale behind the use of short dental implants is that the crestal portion of the implant body is the most involved in load bearing, whereas very little stress is transferred to the apical portion. ${ }^{5}$ Most endosteal dental implants are fabricated from alloyed or pure titanium with a modulus of elasticity (stiffness) approximately five times greater than dense cortical bone. A basic mechanical principle states that when two materials of different moduli are placed together with no intervening material and one is loaded, a stress concentration can be observed where the two materials first come into contact. ${ }^{10}$ Based on this principle, an increased length would simply improve primary stability of the implant during initial placement and enhance osseointegration. On the other hand, a wider diameter implant would increase not only 
primary stability but also the functional surface area at the crestal bone level, and thus lead to a better distribution of occlusal forces to the surrounding bone. Therefore, short wide-diameter implants should bear functional stresses as effectively as longer implants. ${ }^{9}$ These findings were confirmed using finite elemental analysis (FEA) by various researchers. It was demonstrated by FEA that horizontal and vertical occlusal forces placed on implants were distributed primarily in the crestal bone, rather than along the entire implant/bone interface. ${ }^{11}$ Also, FEA indicates that maximum bone stress is practically independent of implant length. ${ }^{12}$

\section{Anatomical Considerations for Implant Placement}

Adjacent anatomical structures are key factors in the planning and placement of dental implants. In both the maxilla and mandible, the height and width of available (residual) alveolar bone is crucial; when a relative insufficient bone is present, implant positioning may be suboptimal to compensate for the lack of sufficient bone. In addition, bony undercuts can lead to perforation of the cortical bone during preparation of the osteotomy and/or lead to suboptimal implant placement, and the apices of teeth may be very close to a proposed osteotomy site. Beyond this, there are specific anatomical factors to consider for each arch.

\section{Maxillary Arch}

Anatomical considerations in the maxilla include the maxillary sinus posteriorly and, much less frequently, the nasal floor anteriorly and the nasopalatine canal. With respect to the maxillary sinus, iatrogenic sinus perforation can occur if implants are too long for the available bone height, and this has been found to potentially result in a higher failure rate for implants. When standard-length implants are to be used in these situations, a ridge augmentation bone graft may be required to increase the height and volume of bone. Note that ridge augmentation may also be required to accommodate an implant's diameter along its length. Placing implants in the posterior maxillary region is particularly challenging, given the possibility of sinus problems, insufficient space between the arches and previous bone resorption. In the maxillary sinus region, enlargement of the maxillary sinus (pneumatization) $)^{13}$ also occurs due to bone loss from the internal aspect of the sinus walls following tooth loss (or due to aging). Grafting the floor of the maxillary sinus was the most common surgically modality for correcting this inadequacy. This technique, first published in 1980 by Boyne and James and subsequently modified by other clinicians can result in increased bone height. ${ }^{14}$ An invasive, external sinus lift procedure may be required to prevent implant penetration through the sinus membrane into the floor of the sinus or the implant being introduced into the maxillary sinus. Alternatively and less invasively, an internal sinus lift can often be performed with an osteotome. It is more likely for this to be possible with use of a short implant. An atrophic maxilla along with poor bone quality is always a risk factor for placing implants. ${ }^{6,15}$ On rare occasions, the nasopalatine canal region is problematic. Cases have been reported where a block bone graft is placed over the nasopalatine area after the soft tissue is pushed back into the canal, resulting in successful placement of implants without the loss of sensation. Extra care is required to avoid perforating the nasopalatine canal when placing immediate implants in any patient where the osteotomy site is in proximity to the canal position.

\section{Mandibular Arch}

The inferior dental canal in the resorbed mandible is by far the most frequent and important anatomical consideration in the mandible. If unaddressed, this anatomical problem can result in impingement or penetration of the inferior dental canal with resulting pain and sensory impairment as well as arterial damage. Solutions to these anatomical challenges prior to placement of standard-length implants include ridge augmentation, bone grafts and the transpositioning of the inferior dental nerve and artery. The inferior dental canal may also be positioned more superiorly in some patients. In the interforamenal area, the amount of bone resorption and anatomy of the mental nerve must be considered to avoid perforation of the cortical plate or impinging on the mental nerve. Less frequent considerations include anterior looping of the mental nerve and the lingual foramena may present challenges. In assessing patients and considering implant length and position, the individual differences may include the presence of bifid mandibular canals and accessory mental foramena. Reconstruction of the atrophic mandible using short implants without augmentation procedures yielded, after more than 10 years of follow-up, a cumulative implant survival rate of $92.3 \%{ }^{16}$

\section{Short Implants vs Adjunctive Procedures}

In some patients, regular-length implant placement must, as discussed above, be preceded by adjunctive procedures in the resorbed mandible or maxilla. The adjunctive procedures described above to augment bone and deal with anatomical structures have their own challenges. Bone grafting, osseodistraction, nerve repositioning and sinus lifts are invasive procedures. In addition, they add complexity and increase the number of surgical phases required for implant therapy. They are also not without the possibility of 
complications resulting from these procedures themselves. Bone grafting, whether autogenous or allogenous, carries with it a risk of complications that include the harvesting procedure itself (for autogenous grafts) and the possibility of graft infection, poor flap closure, dehiscence and resorption of the graft.

Guided bone regeneration using membranes and autogenous bone grafting is much less commonly performed in these situations and has also been found to result in complications, including exposure of the membrane and wound dehiscence.

Short implants are a much less complex and less invasive treatment than placement of longer implants in clinical sites where prior adjunctive ridge augmentation, localized bone grafting, inferior mandibular nerve repositioning or maxillary sinus elevation would be required. They also result in the removal of less bone than with longer implants and are less invasive compared to these and therefore probably less traumatic.

Short implants simplify treatment in the posterior resorbed maxilla and mandible and reduce the number of situations where adjunctive therapy is required. Short implants can also be placed if previous bone graft resorption has occurred at a site intended for longer implants. They also remove the need for cantilevers that might otherwise be required to avoid placing implants in an area with resorbed bone and that are associated with a higher failure rate.

\section{Factors in the Success of Short Implants}

The viability and high success rates seen with short implants (while in the natural dentition a similar 'crown-to-root' ratio would be predictive of failure) can be explained by osseointegration, the crown-implant ratio, the macrogeometric design of the implant, as well as physics and the distribution of forces.

\section{Osseointegration}

The interface between the endosseous root-form dental implant and the surrounding bone involves the process of osseointegration whereby the bone grows around and adheres to the surface of the implant. The bone-implant connection is direct, and no periodontal ligament exists. When osseointegration is complete, the result is a stable, rigid ankylosed connection between the implant and bone that will support the occlusal load, provided the osseointegrated surface is adequate. In contrast, in a natural tooth the root is attached to the alveolar process by the periodontal ligament and, additionally, the root-form is typically conical. This results in the unfavorable distribution of forces and eventually failure in short crown-to-root ratio situations.

\section{Crown-to-Implant Ratio vs Crown-to-Root Ratio}

Misch states that the crown-to-implant ratio should not be considered the same way as a crown-to-root ratio. ${ }^{17}$ In treatment planning of conventional fixed prosthodontic restorations using natural teeth as abutments, Ante's law states that 'the combined pericemental area of all of the abutment teeth should be equal to or greater than the pericemental area of the teeth to be replaced.' As a result, clinicians would treatment plan implant supported restorations with long machined fixtures in an effort to follow Ante's law. It soon became evident, though, that a crown-to-implant ratio of 1:1 was extremely successful and completely acceptable. However, in the posterior regions of the mouth, there is usually natural resorption of the alveolar ridge as a result of prolonged edentulism that leads to an amplified inter-arch distance. The consequent limited available bone leads the practitioner to consider the option of short implants. This would lead to a poorer 1:2 implant-to-crown ratio. Surprisingly, the improvements of surfaces and implant systems, along with proper force orientation and load distribution have allowed such ratios to be applied with success under certain criteria. ${ }^{8}$

\section{Macrogeometric Design and Diameter of Short Implants}

The macrogeometric design and diameter of implants have been found to be relevant for applied forces and stress. Logically, increase in diameter increases the surface area of the bone-implant interface and thus an increase in diameter could compensate for decreased length. There is, of course, an anatomical limit to how much the diameter of implants can be increased. Where there is compromised bone height where short implants seem to be the only solution, to compensate for a shorter length, a wider-diameter implant $(5 \mathrm{~mm})$ can be used. In fact, the use of a $5 \mathrm{~mm}$ diameter implant that is $6 \mathrm{~mm}$ long increases the surface area available to contact the bone similar to that of a $3.75 \mathrm{~mm}$ diameter implant that is $10 \mathrm{~mm}$ in length. To reduce the risk of failure of endosseous implants used in the posterior regions, widediameter implants have been suggested. ${ }^{18}$

When studying the influence of diameter other factors that increase surface area of the bone-implant interface include the presence of rough surface areas (e.g. SLA, TiUnite ${ }^{19}$ and plateaus (fins). Advantages of rough implant surfaces include increased contact area to offer better mechanical stability between bone and implant immediately following insertion provides surface configuration that properly retains the blood clot, stimulates the bone healing process. Himmlova et al found that an increase in the diameter of implants was associated with reduced stress at 
the implant neck and good distribution of force compared to increases in implant length. ${ }^{20}$ Hagi et al studied implants $7 \mathrm{~mm}$ or less in length in partially edentulous patients and concluded that the surface geometry of the implants was a major factor in success rates for these implants; also they found that threaded implants had a higher failure rate and sintered porous-surfaced short implants were successful. ${ }^{21}$

With respect to the macrogeometric design of implants, long and short alike, it is known that an implant's contour can significantly influence load transfer and bone failure rates. Bozkaya et al researched the differences in occlusal loading and bone failure for implants with different macro designs but similar size. These differences included the thread profiles and the crest module shapes. To test the implants, moderate and high loads simulating occlusal loads in function and parafunction were placed on the single-tooth implant abutments and the total overloaded bone estimated. While the thread design and crest module shape were not relevant for overloading when moderate occlusal loads were applied, once high occlusal loads $(\geq 1000 \mathrm{~N})$ were applied, differences in the area of overloaded bone were found, depending on the macrogeometric design of the implant. ${ }^{22}$

\section{Physics and the Distribution of Forces}

Physics and the distribution of forces self-evidently dictate that the majority of the force and compression at the implantbone interface occurs in specific areas of its length; the middle of the implant's length is a wasted area with respect to the distribution of forces. Logically, therefore, it is the availability of sufficient area where the forces are distributed that is important, rather than the total length of an implant - the length of the middle portion of the implant, where forces are not distributed, is not relevant. Nonetheless, if implants are short and also of very narrow diameter, they are at risk for implant failure, implant component fracture or other complications with repeated application of forces, particularly in the posterior maxilla or mandible. On the other hand, the area exposed to force and compression can be increased by roughening the surface and/or employing a macrogeometric design that provides for a favorable shape. A roughened surface also increases the area for ankylosis to bone (and therefore the surface area in intimate contact with bone).

\section{DISCUSSION}

Perhaps clinicians should reconsider the way they view placement of short implants. Short implants can be a very effective substitute to sinus grafting. However, there are several guidelines/suggestions that should be taken into consideration. The most important aspect of implant treatment with short implants is 'case selection.' For example, it would seem practical to follow a two-stage implant surgery approach when placing short implants, since this approach has been associated with higher success rates with short implants. It may also prove wise to avoid placing short implants in single molar cases in free-end situations but rather splint them to an additional implant (preferably longer), especially when placed in soft bone; type III or IV. Soft bone is alone a risk factor, so coupling it with a single short implant only augments the potential problem. Most implant failures can be attributed to poor bone quality. Occlusion is a crucial factor in longevity of implant treatments. Maximal occlusal forces applied and tolerated vary greatly according to implant position in the arch, parafunctional habits of the patient (bruxism/clenching), and nature of the opposing dentition. Biomechanical overload can easily be rendered with high bending moments, unfavorable force distributions, and increased force magnitude commonly seen in the posterior region of the mouth. Overloading may lead to loss of osseointegration and fracture of the implant or the superstructure. When placing short implants, it has also become evident from the literature that compensating with wider implants is the most sensible approach. $^{18}$

\section{CONCLUSION}

Shorter implants are less invasive than placing longer implants with adjunctive therapy to create adequate bone. This results in less pain and discomfort for patients and a shorter healing period and overall treatment time. In addition, dental implants offer practical and successful alternatives to treatments that may otherwise result in the destruction of healthy tooth structure, such as with the placement of a fixed partial denture where the abutment teeth are unrestored or minimally restored. In this situation, the ability to offer a short implant may result in the patient accepting what would be regarded as better treatment compared to the use of healthy tooth structure for fixed partial dentures. Cost is also a major consideration for many patients. While the surgical and restorative phases of implant therapy are expensive, adjunctive procedures such as bone grafting and ridge augmentation can increase the cost that must be borne by the patient. As the evidence collects for the efficacy and success of short implants in suboptimal or difficult sites, the inevitable clinical question arises, 'why not use short implants in regular or optimal sites?'

\section{REFERENCES}

1. Chizolini EP, Rossi AC, Freire AR, Perussi MR, et al. Short Implants in Oral Rehabilitation. RSBO 2011;8(3):329-334.

2. Grant BN, Pancko FX, Kraut, RA. Outcomes of placing short dental implants in the posterior mandible: a retrospective study of 124 cases. J Oral Maxillofac Surg 2009;67:713-717. 
3. Renouard F, Nisand D. Impact of implant length and diameter on survival rates. Clin Oral Impl Res 2006;17(Suppl 2):35-51.

4. Hasan I, Bouravel C, Mundt T, Heinemann F. Biomechanics and Load Resistance of Short Dental Implants: A Review of the Literature. ISRN Dentistry 2013April:1-5.

5. Annibali S, Cristalli MP, Dell'Aquila D, Bignozzi I, et al. Short dental implants: a systematic review. J Dent Res 2012;91: 25-32.

6. Telleman G, Raghoebar GM, Vissink A, den Hartog L, et al. A systematic review of the prognosis of short $(<10 \mathrm{~mm})$ dental implants placed in the partially edentulous patient. J Clin Periodontol 2011;38:667-676.

7. Lai HC, Si MS, Zhuang LF, Shen H, et al. Long-term outcomes of short dental implants supporting single crowns in posterior region: a clinical retrospective study of 5-10 years. Clin Oral Impl Res 2013;24(2):230-237.

8. Galvao FFSA, Almeida-Junior AA, Faria-Junior NB, Caldas SGFR, et al. Predictability of short dental implants: a literature review. RSBO 2011;8(1):74-80.

9. Raviv E, Turcotte A, Harel-Raviv M. Short dental implants in reduced alveolar bone height. Quintessence Int 2010;41(7):41-45.

10. Misch CE. Short dental implants: a literature review and rationale for use. Dent Today 2005;77:64-68.

11. Fugazzotto PA, Beagle JR, Ganeles J, Jaffin R, et al. Success and failure rates of $9 \mathrm{~mm}$ or shorter implants in the replacement of missing maxillary molars when restored with individual crowns: preliminary results 0 to 84 months in function: a retrospective study. J Periodontol 2004;75:327-332.

12. Malo P, Nobre MA, Rangert B. Short implants placed one-stage in maxillae and mandibles: a retrospective clinical study with 1 to 9 years of follow-up. Clin Implant Dent Relat Res 2007; $9(1): 15-21$
13. Lops D, Bressan E, Pisoni G, Cea N, et al. Short implants in partially edentulous maxillae and mandibles: A 10 to 20 years retrospective evaluation. Int J Dent 2012;1-8.

14. Wallace SS, Froum SJ. Effect of maxillary sinus augmentation on the survival of endosseous dental implants: a systematic review. Ann Periodontal 2003;8(1):328-343.

15. Cantalejo EM, Dorado CB, Alvarez MC, Caliz FF, et al. Metaanalysis on the survival of short implants. Med Oral Patol Oral Cir Bucal 2011;16(4):546-551.

16. Karthikeyan I, Desai SR, Singh R. Short implants: A systematic review. J Indian Soc Periodontol 2012;16(3):302-312.

17. Birdi H, Schulte J, Kovacs A, Weed M, et al. Crown-to-implant ratios of short-length implants. J Oral Implantol 2010;36(6): 425-433.

18. Morand M, Irinakis T. The challenge of implant therapy in the posterior maxilla: providing a rationale for the use of short implants. J Oral Implantol 2007;33(5):257-266.

19. Van Assche N, Michels S, Quirynen M, Naert I. Extra short dental implants supporting an overdenture in the edentulous maxilla: a proof of concept. Clin Oral Impl Res 2012;23:567-576.

20. Himmlova L, Dostalova T, Kacovsky A, Konvickova S. Influence of implant length and diameter on stress distribution: a finite element analysis. J Prosthet Dent 2004;91:20-25.

21. Hagi D, Deporter DA, Pilliar RM, Arenovich T. A targeted review of study outcomes with short $(\leq 7 \mathrm{~mm})$ endosseous dental implants placed in partially edentulous patients. J Periodontol 2004;75(6):798-804.

22. Bozkaya D, Moftu S, Muftu A. Evaluation of load transfer characteristics of five different implants in compact bone at different load levels by finite elements analysis. J Prosthet Dent 2004;92:523-530. 